\title{
Development of a novel flapping mechanism with adjustable wing kinematics for micro air vehicles
}

\author{
A. T. Conn, S. C. Burgess \& R. A. Hyde \\ Department of Mechanical Engineering, University of Bristol, \\ Bristol, UK
}

\begin{abstract}
There is currently significant interest in developing micro air vehicles (MAVs). Insects provide a natural blueprint for such vehicles and can outperform current man-made miniature flyers through the exploitation of low Reynolds number aerodynamics. However, designing a flapping mechanism that replicates the complex wing kinematics of insects is a demanding task, due to limitations in current actuation technology and the miniature scale of MAVs. The majority of current MAV flapping mechanisms produce constrained wing kinematics and therefore have little capacity for replicating insect flight manoeuvres and hence controlled flight. This paper presents a novel mechanism that produces partially constrained insect inspired wing motion with adjustable kinematic parameters. The parallel crank-rocker mechanism can modulate the phase difference between the rocker links to achieve this and hence produce controllable flight. Its overall development is described and the wing kinematics it produces are compared with those of a hawkmoth.

Keywords: micro air vehicles, insect flight, wing kinematics, flapping wing mechanism, biomimetic, bio-inspired, unsteady aerodynamics.
\end{abstract}

\section{Introduction}

\subsection{A Brief History of MAVs}

Micro air vehicles (MAVs) are miniature flying craft that are distinguishable from the current smallest flying vehicles such as military unmanned air vehicles and hobby-enthusiast model airplanes and helicopters by their scale, which in 
terms of mass and volume is at least an order of magnitude less. The concept of a MAV was initiated by military strategists, inspired by rapidly advancing technologies such as micro-electromechanical systems (MEMS) and miniature digital cameras [1]. They envisioned low cost, highly miniaturised autonomous robots capable of unheralded levels of reconnaissance, surveillance and hazardous substance detection. A miniature, autonomous flying vehicle also has many civil and commercial applications such as search and rescue, traffic monitoring and air quality sampling.

MAVs are commonly defined by a $15 \mathrm{~cm}$ dimensional limit, instigated by the U.S.A.'s Defense Advanced Research Projects Agency (DARPA), that founded the first MAV research program. There are likely to have been two primary reasons behind this limit. The first is simply due to anthropometrics, since $15 \mathrm{~cm}$ approximately conforms to being "hand-held" and is also suitable for personal storage [2]. The second and more profound reason was based on aerodynamic principles. Having a maximum characteristic length of under $15 \mathrm{~cm}$ coupled with a relatively low flight speed means MAVs are certain to operate in a laminar flow regime with a Reynolds number below $5 \times 10^{5}$, the value commonly attributed to the transition between laminar and turbulent flow for a flat plate [3].

\subsection{Insect inspired flapping MAVs}

A MAV can have any type of propulsion system, but currently the three most viable platforms are fixed wing, rotorcraft and flapping wing. Flight performance varies between them but can be characterised as fixed wing MAVs being better suited to outdoor operations while rotorcraft and flapping wing MAVs' abilities to hover and vertically take-off and land (VTOL) allow them to manoeuvre through cluttered indoor environments. If the desire is to create a MAV capable of flying indoors, at first it appears the basic flight performance (flight speed, hover capabilities etc.) of rotorcraft and flapping wing MAVs are similar. Flapping wing MAVs, however, have a much greater potential for extreme manoeuvrability and high payload capacity. This potential is apparent in a tangible sense by the fact that millions of natural flapping wing "MAVs" already exist in the form of insects and small birds, who regularly exhibit exceptional flight agility.

Insects, in particular, achieve this outstanding flight performance by exploiting unsteady aerodynamic mechanisms that exist at the low Reynolds number flow regime they share with MAVs. This has lead to great interest in mimicking the complex wing kinematics of insects with the explicit aim of replicating the same unsteady aerodynamic phenomena. This paper details research into the development of an insect inspired flapping mechanism for application to a highly manoeuvrable, hover-enabled MAV. It should be noted that flapping MAVs that have not copied wing kinematics found in nature have been successfully flown but with limited hovering ability e.g. Jones and Platzer [4]. 


\section{Insect inspired biomimetic design}

\subsection{Unsteady aerodynamics}

The understanding of the theory behind the unsteady aerodynamic mechanisms employed by insects has significantly improved over the last two decades, but is still subject to some uncertainty and contradiction. Also, not all insects utilise the same mechanisms, in part due to differing morphologies, wing kinematics and Reynolds numbers. While both the smallest and largest insects fly in a laminar flow regime, they span the transition from viscosity dominated flow (Re $\left.=10^{0}-10^{2}\right)$ to inertia dominated flow $\left(\operatorname{Re}=10^{2}-10^{4}\right)$ respectively $[5,6]$. It is likely a flapping MAV will operate in inertia dominated flow and so the unsteady aerodynamic mechanisms of interest are those thought to be employed by larger insects:

- Leading edge vortex (LEV) and dynamic stall

- Wing rotation force - Kramer effect

- Wake recapture

- Virtual mass

A full description of how these mechanisms augment lift from insect wings will not be given, as they have been subject to numerous review studies (e.g. Lehmann [7]; Sane [8]). The two most important unsteady mechanisms in terms of lift are the LEV and the timing-dependent rotational force thought to be produced through the Kramer effect [8]. It has been shown with a mechanical hawkmoth model that a LEV may account for lift equal to two thirds of a hawkmoth's body weight [9]. Dickinson et al. [10] found that the rotational force contributed $35 \%$ of the lift produced by a similar flapping mechanical model and that this value was extremely sensitive to the timing of the rotation.

\subsection{Mimicry of insect flight}

From the perspective of designing a flapping MAV, replicating the unsteady aerodynamic mechanisms utilised by insects appears to be a difficult task. It is simply not feasible to reverse engineer an insect's thorax, which contains its flight muscles and wing joint, so the best approach is to mimic just the insect's wing kinematics and not its thorax morphology. However, there is great variation of wing kinematics amongst insects (even for the same species), suggesting they are altered to suit specific aerodynamic demand [11]. This has caused a gap in knowledge over what the optimal wing kinematics are for a particular wing and hence uncertainty over which kinematical features each unsteady aerodynamic mechanism is dependent on. Therefore mimicking insect wing kinematics is likely only to provide the starting point for flapping MAV design, with aerodynamic experimentation leading to wing kinematics optimisation currently a necessity for optimal performance.

Apart from their wing kinematics, insects possess other features pertinent to biomimicry. Insect wings are complex, lightweight structures that possess several common features to maximise the production of lift and thrust. The form 
and orientation of wing veins in particular create regions of high stiffness and of high compliance to ensure the wing reacts favourably to local airflows throughout the entire wing stroke. Another optimal design feature is insects' use of resonance and elastic storage. The insect's thorax is a mechanically resonant system that incorporates highly elastic elements, composed of resilin, which store kinetic energy at the end of each upstroke and downstroke and release it during the half-stroke. Creating a material that matches resilin's elastic properties is not currently feasible, yet the principle of elastic storage remains a hugely beneficial method of minimising power expenditure.

\subsection{Insect wing kinematics}

It was outlined in the previous section that there is great variation of wing kinematics amongst insects and therefore it is necessary to generalise them for application to a flapping MAV mechanism. Wing strokes involve two translatory stages (downstroke and upstroke) interspersed by two short rotational stages (supination and pronation), at which point the wing reverses direction. The motion of the wing through these stages is described using a set of kinematic parameters, of which typical values are given in Table 1. Producing a flapping mechanism that matches the kinematic parameters of insects should produce the desired unsteady aerodynamic mechanisms. However, if these parameters cannot be dynamically adjusted then the MAV will have no capacity for flight stability or manoeuvring and will have no chance of staying airborne. Detailed knowledge of insect flight control is currently lacking, but it is possible to classify which kinematic parameters need to be adjusted to perform certain manoeuvres (as shown in Table 2). It should be noted that a MAV might not need to adjust all its kinematic parameters for controlled flight, and it may be beneficial for the design of the flapping mechanism to constrain certain parameters.

Table 1: $\quad$ Typical values of kinematic parameters for insects.

\begin{tabular}{|c|c|c|}
\hline Side View of Insect & Kinematic Parameter & Typical Value \\
\hline \multirow{7}{*}{$\begin{array}{l}\text { Example } \\
\text { trajectory }\end{array}$} & Wingtip trajectory & (shown on diagram) \\
\hline & Angle of attack, $\alpha$ & $30^{\circ} \quad[6]$ \\
\hline & Stroke amplitude, $\Phi$ & $120^{\circ}[12]$ \\
\hline & Wing beat frequency, $n$ & $\begin{array}{c}20-40 \mathrm{~Hz} \\
\text { for large insects [12] } \\
\left(n \propto \mathrm{m}^{-1 / 4}\right)\end{array}$ \\
\hline & Stroke timing, $d / u$ & $1.0-1.1[13]$ \\
\hline & Stroke plane angle, $\beta$ & $\begin{array}{c}10^{\circ} \rightarrow 50^{\circ} \quad[6] \\
\text { (flight speed: } 0 \rightarrow \text { max.) }\end{array}$ \\
\hline & Body angle, $\chi$ & $\begin{array}{c}50^{\circ} \rightarrow 10^{\circ} \\
\text { (flight speed: } 0 \rightarrow \text { max.) }\end{array}$ \\
\hline
\end{tabular}


Table 2: Kinematic parameter modulation required for performing manoeuvres.

\begin{tabular}{|c|c|c|c|c|c|}
\hline Manoeuvre & Roll & Pitch & Yaw & Kinematic Parameter & $\begin{array}{c}\text { Wing } \\
\text { Balance }\end{array}$ \\
\hline \multirow{3}{*}{$\begin{array}{c}\text { Forward } \\
\text { acceleration }\end{array}$} & & $(\mathrm{X})$ & & Stroke plane angle [6] & Symmetric \\
\hline & & $(\mathrm{X})$ & & Stroke amplitude [6] & Symmetric \\
\hline & & $(\mathrm{X})$ & & Wing beat frequency [14] & Symmetric \\
\hline $\begin{array}{c}\text { Nose up/ } \\
\text { nose down }\end{array}$ & & $\mathrm{X}$ & & Stroke timing [14] & Symmetric \\
\hline \multirow{2}{*}{$\begin{array}{c}\text { Vertical } \\
\text { acceleration }\end{array}$} & & & & Stroke amplitude & Symmetric \\
\hline & & & & Wing beat frequency & Symmetric \\
\hline \multirow{2}{*}{$\begin{array}{c}\text { Lateral } \\
\text { acceleration }\end{array}$} & $\mathrm{X}$ & & & Stroke amplitude [6] & Asymmetric \\
\hline & $\mathrm{X}$ & & & Angle of attack [6] & Asymmetric \\
\hline \multirow{2}{*}{ Flat Turn } & & & $\mathrm{X}$ & Angle of attack [10] & Asymmetric \\
\hline & & & $\mathrm{X}$ & Stroke timing [14] & Asymmetric \\
\hline \multirow{2}{*}{$\begin{array}{c}\text { Banked } \\
\text { turn }\end{array}$} & $\mathrm{X}$ & & $\mathrm{X}$ & Stroke amplitude [6] & Asymmetric \\
\hline & $\mathrm{X}$ & $\mathrm{X}$ & $\mathrm{X}$ & Stroke plane angle [6] & Asymmetric \\
\hline
\end{tabular}

\section{Selection of flapping mechanism}

\subsection{Actuation system}

The extreme mass and volume constraints on-board MAVs, as well as a limited power supply, require them to be highly integrated systems, much like their natural inspiration. As a result, even when considering just the flapping mechanism it is critical to include the actuation devices at an early stage of the design process. Assessing the actuation technologies currently available, it is clear there are no currently available products that come close to matching muscle's extremely high strain output (likely to be essential for a compact mechanism to produce a large enough stroke amplitude). Piezoelectric ceramic benders possess excellent power densities and frequency ranges, but require very high activation voltages to achieve a large enough strain. This is major issue since a MAV's on-board power supply will be extremely limited. A new class of similar smart materials called electro-active polymers (EAPs) may bridge the gap and provide high strain at low voltages but their development is still in its infancy. Brushed DC motors provide a rotary alternative and have a high power density since they do not need bulky driver electronics.

\subsection{Mechanism classification}

As well as the previously stated kinematic parameters, the limitations of available actuation devices add further design specifications to the flapping mechanism. This can be generalised to a linear input mechanism needing high amplification ratios (maximise the input displacement) while a rotary input mechanism requires linkages that can convert rotary motion into controlled 
flapping. Also, since linear actuators can usually modulate their output displacement, they can drive under-constrained mechanisms. Rotary actuators, however, need to continuously perform full revolutions so the corresponding wing motion is likely to be constrained to a single path. The general effect the level of constraint has on a mechanism's performance is described in Figure 1.

Increasing control complexity (more input D.o.F.) and number

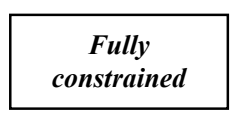
of adjustable kinematic parameters

\section{Decreasing mechanical complexity and wing motion repeatability}

Figure 1: Relationship between level of constraint and performance/complexity.

Since increasing mechanical complexity generally adds more weight while greater control complexity does not, an under-constrained mechanism appears to be the optimum solution. Yet when the limited strain output of available linear actuators is considered, the mechanical realisation of such a mechanism becomes less viable. This is reflected by the flapping mechanisms that have thus far been developed for application to an insect inspired MAV, a selection of which are listed in Table 3. The table summarises each mechanism's kinematical properties in terms of its mechanical complexity (i.e. number of links and joints) and capability for controlling wing kinematics. Note that none of the mechanisms in Table 3 can adjust the stroke plane angle and it is assumed that all can modulate the wing beat frequency, which is an actuation issue.

The parallel four-bar mechanism has the greatest potential for controlled flight as it can dynamically adjust its wing kinematics [15]. Correspondingly, it also has the highest number of input D.o.F and thus control complexity. The other existing mechanisms developed all produce constrained wing kinematics from a single rotary input. All the mechanisms can approximately replicate the kinematic parameters listed in Table 1, although some have simplified the flapping trajectory to a single plane. However, only mechanisms with freely adjustable kinematic parameters can enact the manoeuvres described in Table 2, which are essential for controllable flight.

An alternative solution has been developed to the current flapping mechanisms that uses parallel crank-rockers and is shown in Table 3 in bold. It utilises a rotary input for the main drive and two subsidiary inputs that may be linear or rotary. Since the main drive is rotary, a low voltage DC motor can be used. It requires a comparatively high number of links and joints, but because the links are orientated in the same plane as the wing's stroke plane it is extremely compact. Its main advantage, however, is the ability to adjust each wing's angle of attack asymmetrically. This is achieved by reciprocating two phased rockers per wing. When the phase difference between each rocker is modulated, the angle of attack is altered. This allows it to accelerate laterally and perform flat turns as well as maximising the timing-dependent wing rotation force produced via the Kramer effect. 
Design and Nature III: Comparing Design in Nature with Science and Engineering 283

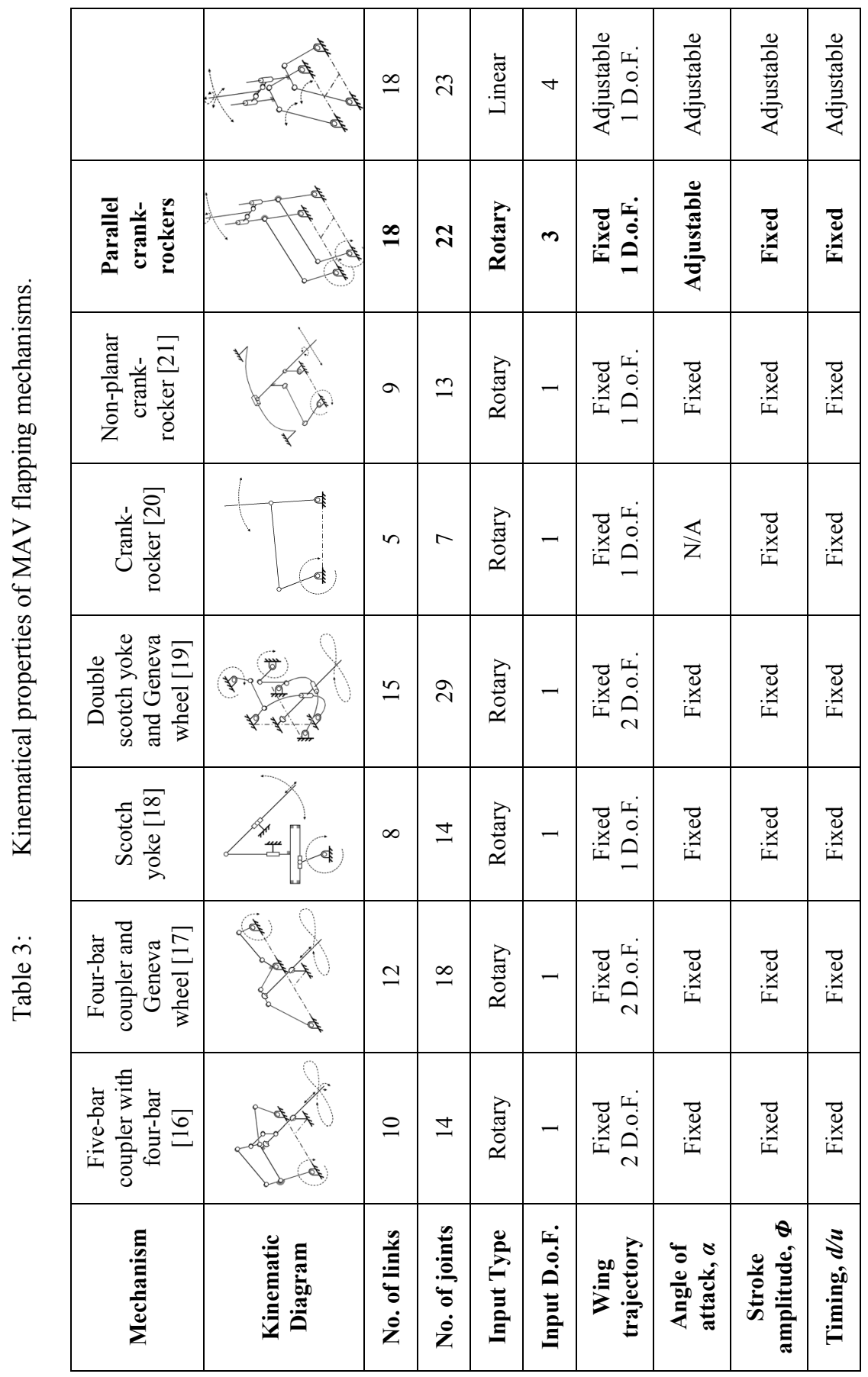




\section{Design of parallel crank-rocker mechanism}

The parallel crank-rocker mechanism forms the basis of an experimental test-rig that produces adjustable wing kinematics that is being manufactured at the University of Bristol. The test-rig has been designed to be fabricated at nearMAV scale and will be used to test both the chosen mechanism's feasibility and for wing kinematics optimisation. The optimisation should help eliminate gaps in knowledge linking wing kinematics and unsteady aerodynamic lift production. The mechanism's link ratios were selected after kinematic analysis, with a final ratio of 1:3.8:1.3:4. This ratio produces a stroke amplitude of $102^{\circ}$ and a stroke timing $(d / u$ ratio) of 1 , which match the general kinematic parameter values given in Table 2 and suggests the unsteady aerodynamic mechanisms should be produced. A comparison of the wing kinematics produced by the mechanism with those of a hawkmoth is shown in Figure 2a. The mechanism's wing kinematics are similar to the hawkmoth's, although the stroke amplitude and $d / u$ ratio are less and the wing motion is restricted to a single plane. The adjustable phase difference between each wing's rockers directly controls the wing angle of attack, and can be adjusted by slowing one crank relative to the other.

Dynamic analysis was undertaken using a combined physical and electrical model created in the Simulink ${ }^{\circledR}$ SimMechanics environment. The results show that mimicking insects' use of elastic storage by attaching springs to the rockers can lower the work done per wing stroke by over $50 \%$ as shown in figure $2 \mathrm{~b}$, even when operating outside of the system's resonant frequency. A geared Maxon RE10 miniature brushed DC motor (7 g mass) was selected and is expected to drive the $75 \mathrm{~mm}$ long wings at a wing beat frequency of $31.7 \mathrm{~Hz}$. Figure 3 shows an annotated CAD view of the final assembly, which has an estimated weight of $62.6 \mathrm{~g}$ with major dimensions of $25 \times 29 \times 62.75 \mathrm{~mm}$. The parts are predominantly composed of aluminium, although certain parts were fabricated using brass due to its availability in lengths of miniature cross-section.

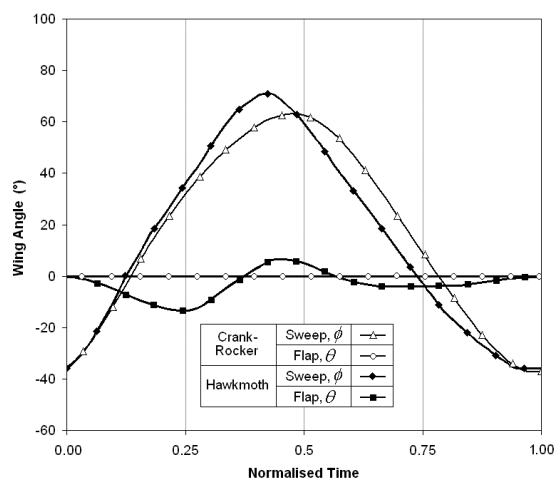

(a)

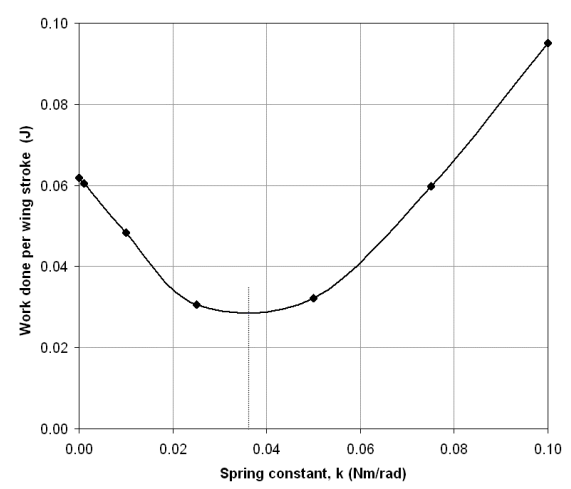

(b)

Figure 2: (a) Comparison of wing kinematics where the sweep and flap angles describe the wing trajectory relative to the stroke plane [11]; (b) Effect of elastic storage on wing stroke work (optimum spring constant indicated by broken line). 


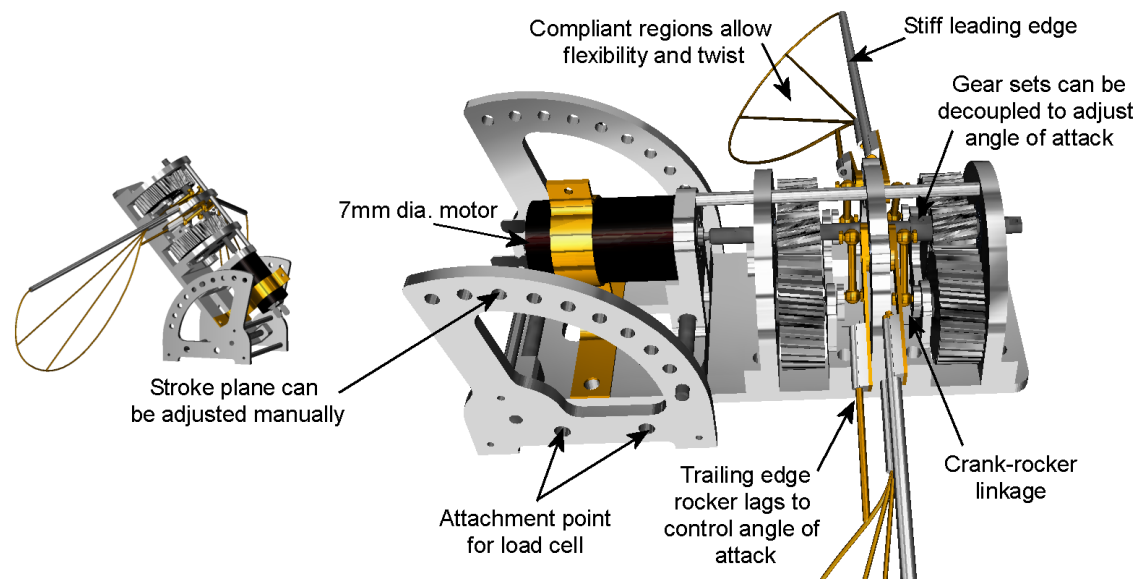

Figure 3: CAD view of mechanism test-rig assembly with key features labelled.

\section{Conclusions}

Insect inspired MAV flapping mechanisms are required to produce complex wing trajectories while possessing compact and lightweight components. A key performance factor in insect flight is the adjustability of wing kinematics, as it is a necessity for controlled flight. Selection of a flapping mechanism should take into account the potential degree of adjustability. The parallel crank-rocker mechanism presented in this paper produces planar wing strokes with a fully adjustable angle of attack, a key kinematic parameter. When integrated with a separate stroke adjustment mechanism this should allow for flight stability and manoeuvring capabilities sufficient for controlled flapping flight.

\section{References}

[1] McMichael, J.M. \& Francis, M.S., Micro Air Vehicles - Toward a New Dimension in Flight, DARPA Document, Online. http://www.fas.org/irp/program/collect/docs/mav auvsi.htm

[2] Defense Advanced Research Projects Agency, News Release, Online. www.fas.org/irp/news/1997/b12121997 bt676-97.html

[3] Anderson Jr., J.D., Fundamentals of Aerodynamics, second edition, McGraw-Hill: New York, 1991.

[4] Jones, K.D. \& Platzer, M.F., On the design of micro air vehicles. Design and Nature, eds: C.A. Brebbia, L.J. Sucharov and P. Pascolo, WIT Press: Southampton, pp. 67-76, 2002.

[5] Dudley, R., The Biomechanics of Insect Flight: Form, Function, Evolution, Princeton University Press: Princeton, New Jersey, 2000.

[6] Ellington, C.P., The novel aerodynamics of insect flight: applications to micro-air vehicles. Journal of Experimental Biology, 202, pp. 3439-3448, 1999. 
[7] Lehmann, F.-O., The mechanisms of lift enhancement in insect flight. Naturwissenschaften, 91(3), pp. 101-122, 2004.

[8] Sane, S.P., The aerodynamics of insect flight. Journal of Experimental Biology, 206, pp. 4191-4208, 2003.

[9] Van den Berg, C. \& Ellington, C.P., The three-dimensional leading-edge vortex of a 'hovering' model hawkmoth. Philosophical Transactions of the Royal Society of London, Series B: Biology, 352, pp. 329-340, 1997.

[10] Dickinson, M.H., Lehmann, F.-O. \& Sane, S.P., Wing rotation and the aerodynamic basis of insect flight. Science, 284, pp. 1954-1960, 1999.

[11] Willmott, A.P. \& Ellington, C.P., The mechanics of flight in the hawkmoth, Manduca Sexta. I. Kinematics of hovering and forward flight. Journal of Experimental Biology, 200, pp. 2705-2722, 1997.

[12] Brodsky, A.K., The Evolution of Insect Flight, Oxford University Press, Oxford, 1994.

[13] Ellington, C.P., The aerodynamics of hovering insect flight. III. Kinematics. Philosophical Transactions of the Royal Society of London, Series B: Biology, 305, pp. 79-113, 1984.

[14] Taylor, G.K., Mechanics and aerodynamics of insect flight control. Biological Reviews of the Cambridge Philosophical Society, 76(4), pp. 449-471, 2001.

[15] Avadhanula, S., Wood, R.J., Steltz, E., Yan, J. \& Fearing, R.S., Lift force improvements for the micromechanical flying insect. IEEE Int. Conference on Intelligent Robots and Systems, Las Vegas, Nevada, October, 2003.

[16] Banala, S.K., Karakaya, Y., McIntosh, S., Khan, Z. \& Agrawal, S.K., Design and optimization of a mechanism for out of plane insect wing motion with twist. Proc. of DETC, ASME Design Engineering Technical Conferences, Salt Lake City, Utah, September, 2004.

[17] Żbikowski, R., Galiński, C. \& Pedersen, C.B., A four-bar linkage mechanism for insect-like flapping wings in hover: Concept and an outline of its realisation. Transactions of the ASME: Journal of Mechanical Design, 127, pp. 817-824, 2005.

[18] Tarascio, M.J., Ramasamy, M., Chopra, I. \& Leishman, J.G., Flow visualization of micro air vehicle scaled insect-based flapping wings. Journal of Aircraft, 42(2), pp. 385-390, 2005.

[19] Galiński, C. \& Żbikowski, R., Insect-like flapping wing mechanism based on a double spherical Scotch yoke. Journal of the Royal Society: Interface, 2(3), pp. 223-235, 2005.

[20] Pornsin-Sirirak, N., Tai, Y.-C., Nassef, H. \& Ho, C.-M., Titanium-alloy MEMS wing technology for micro aerial vehicle application. Sensors and Actuators A: Physical, 89, pp. 95-103, 2001.

[21] Burgess, S.C., Alemzadeh, K. \& Zhang, L., The development of a miniature mechanism for producing insect wing motion. Design and Nature II, eds. M.W. Collins \& C.A. Brebbia, WIT Press: Southampton, pp. 237-244, 2004. 